\title{
LOCALIZATION OF SOUND SOURCES DURING FULL SCALE FATIGUE TEST OF THE VERTICAL STABILIZER WITH THE ACOUSTIC HOLOGRAPHY TECHNIQUE
}

\author{
Andrzej Leski ${ }^{1}$, Michal Szmidt ${ }^{1}$, Piotr Synaszko ${ }^{2}$ \\ ${ }^{1}$ Institute of Aviation, Al. Krakowska 110/114, 02-256 Warsaw, Poland \\ ${ }^{2}$ Air Force Institute of Technology, Ks. Bolestawa 6, 01-494 Warsaw, Poland
}

\begin{abstract}
An acoustic holography and its practical applications in engineering began to develop at the end of the 20th century. Currently, this technique is being commonly used to locate sound sources. This paper presents the use of an acoustic camera to locate sound sources during the Full Scale Fatigue Test of the MiG-29 stabilizer. During fatigue tests, the tested structure issues a series of sounds in the form of glitches, creaks or beats. These sounds are typical for a structure subjected to dynamic loading, but they can also be a source of diagnostic information about places of fatigue failures. The paper presents the results of measurements made during the fatigue test. Thanks to the analysis of the measurement results, it was possible to identify areas that are the basic source of sounds.
\end{abstract}

Keywords: acoustic holography, Full Scale Fatigue Test, damage detection, microphone arrays.

\section{INTRODUCTION}

\subsection{Vertical stabilizer Full Scale Fatigue Tests}

Full Scale Fatigue Tests (FSFT) are a common way to prove fatigue life of aeronautical structures and their main components [6,7]. The FSFT of a MiG-29's vertical stabilizer is being currently carried out at the Institute of Aviation as an important part of a research project dedicated to a new composite patch repair technology. The repaired stabilizer is being tested to prove its fatigue resistance. The test stand consists of a quarter of the whole MiG-29 structure as shown below. 


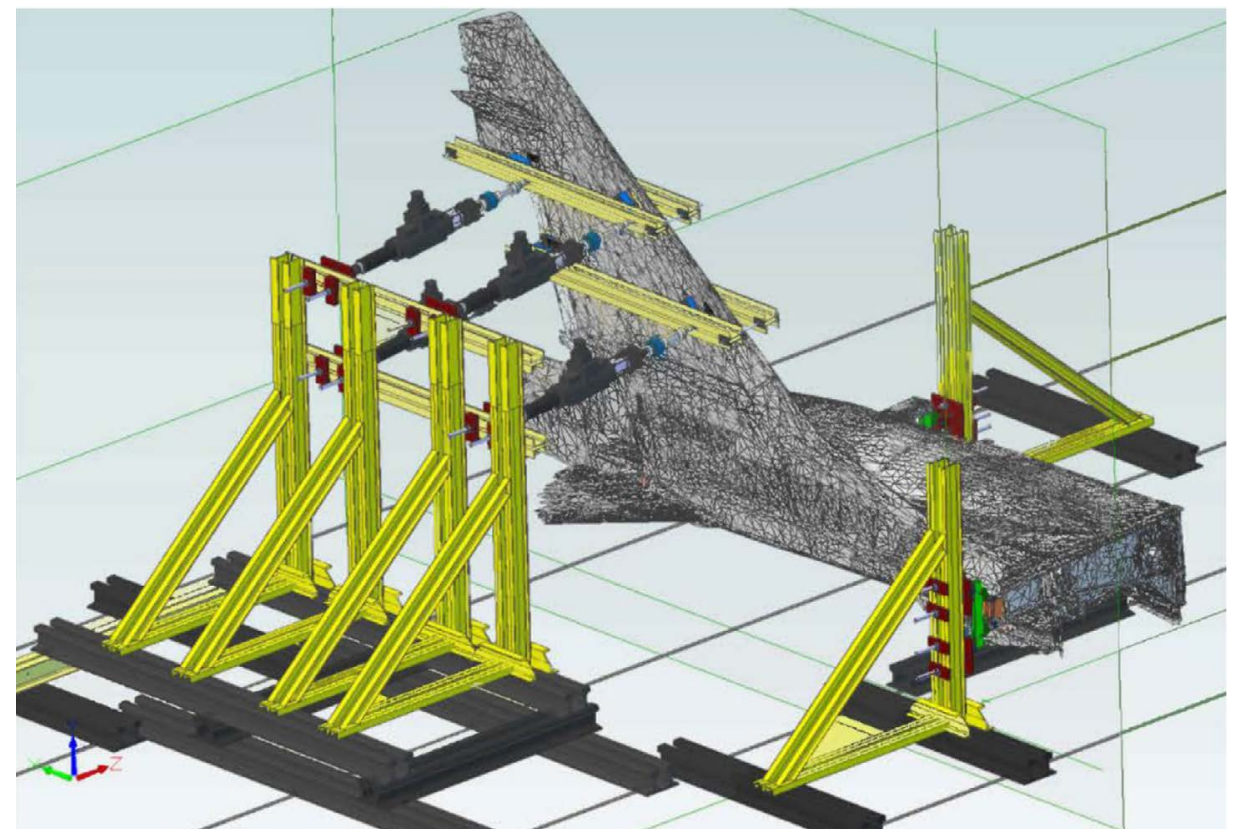

Fig.1 The test rig

A Mig-29 vertical stabilizer consists of a composite top part and a metal lower part. The lower part is integrated with the fuselage. The composite part can be detached. During the test, the area of interest was the top part of the stabilizer with composite patches. A big part of the MiG's structure had to be used to provide proper stiffness at a mounting point. A disadvantage of this solution is the fact that the whole tested structure accumulates fatigue damage and is susceptible to fatigue cracks propagation. In result, the whole structure must be periodically inspected to prevent the rapid crack propagation or unpredicted disintegration.

It is common that a fatigue tested structure emits noise. The noise during FSFT can make an impression even on engineers who are familiar with such tests. The main source of the noise is the buckling of thin-walled parts and friction. Obviously, the presence of noise during fatigue testing can also be used as a diagnostic tool as the presence of cracks or increased wear can result in louder structural noises or just the noises with a changed spectrum. The human ear is invaluable but often unreliable sensor and should be supported with a more sophisticated technique. Such a technique is Acoustic Holography or Acoustic Beamforming 


\subsection{Acoustic Holography versus Acoustic Beamforming}

Started in 90's different methods based on microphone arrays have been used to sound source localization $[1,2,4]$. Two of these, Acoustic Holography and Acoustic Beamforming, are currently most commonly used. Depending on the test object, the sound frequency and the actual environment, engineers have to select either the first method or the latter. What are the differences, advantages and limitations of these methods?

Acoustic Holography (exactly Near-field Acoustic Holography, also called $\mathrm{NAH}$ ) is a technique where the microphone array is placed relatively close to the object - in the near field [5]. The distance between the microphone array and a sound source should be shorter than two wavelengths of the highest frequency. In practice this distance should not exceed $0.5[\mathrm{~m}]$

Near-field Acoustic Holography measures sound pressure by arranging several regularly spaced microphones in a planar array. The sound pressure in the plane is then back-propagated to the actual surface of the object. The spacing between the microphones determines the spatial resolution. If the interested maximum frequency is very high, the spacing becomes very small. This is one of the major disadvantages of NAH.

Acoustic beamforming is a technique where the microphone array is placed in the far field. Numerous microphone configurations are possible in acoustic beamforming arrays. Usually, it is preferred to select a circular array with a pseudo-random microphone distribution. In the far field, sound waves hitting the array are planar waves. Under these conditions, it is possible to propagate the measured sound field directly to the test object. All microphone signals measured by the acoustic beamforming array are added together, taking into account the delay corresponding to the propagation distance. The pressure can be calculated at any point in front of the array, allowing propagation to any type of surface. Acoustic beamforming requires that all data are measured simultaneously. An advantage of acoustic beamforming is the fact that the test object can be larger than the array. Moreover, this technique is relatively fast. A disadvantage of this method is the fact that spacial resolution depends on the antenna diameter $\mathrm{D}$ and the distance between the antenna and the object $\mathrm{d}$ :

$$
\text { spacial resolution }=\lambda \frac{d}{D}
$$

where $\lambda$ is a wave length.

In result, acoustic beamforming is only usable at frequencies above $1000 \mathrm{~Hz}$. Additionally, acoustic beamforming cannot be used to calculate sound power $[3,8]$. 


\section{MEASUREMENTS}

\subsection{Instrumentation}

The FSFT of the MiG-29 vertical stabilizer is conducted inside a laboratory building where there is a low level of continuous noise ( $54 \mathrm{~dB}$ excluding the FSFT noise). The acoustic camera measurements were taken from both sides of the stabilizer (Fig.2 and Fig.3). The microphones array was positioned against the stabilizer at a distance of $1.7 \mathrm{~m}$.

The measurement system consists of:

- portable computer with the LMS Test.Lab software for the signals recording and postprocessing,

- multichannel digital signal recorder LMS SCADAS Mobile,

- circular TL-AHW.16.1 microphones array with 36 G.R.A.S. 40PH ICP microphones and a HD video wide-angle camera placed on a leveled tripod.

Microphones array
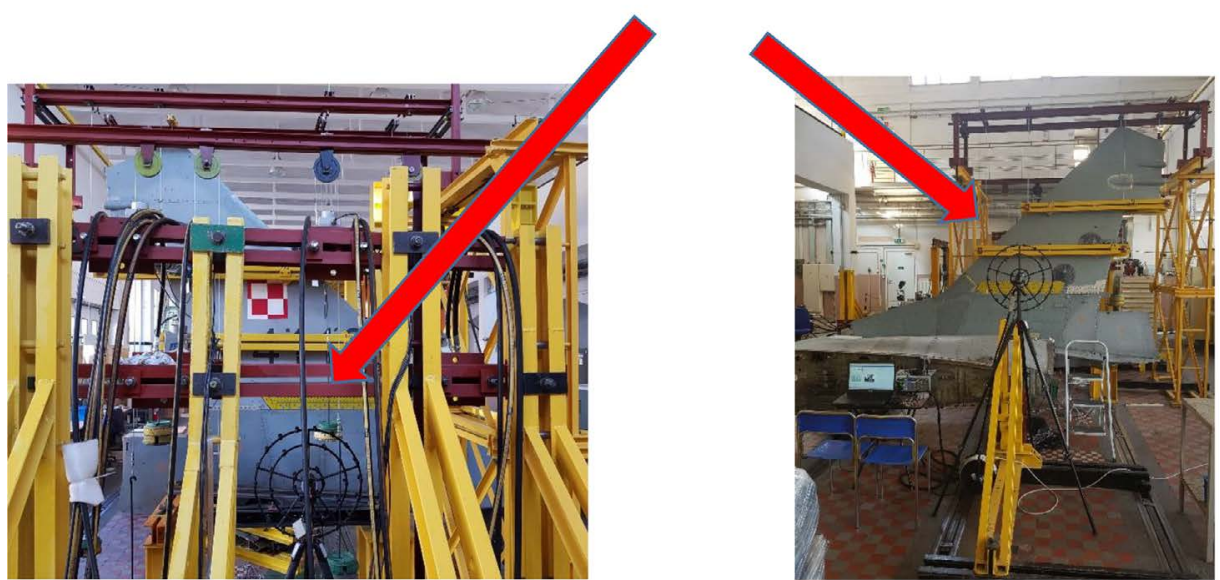

Fig. 2 Microphones array against the vertical stabilizer

A single measurement usually took 40 seconds. During this time all noises were recorded and analyzed. Because the load spectrum for the FSFT consists of a large number different load cases, each measurement was unique in terms of loads and noises.

\subsection{Measurements results}

The maximum recorded frequency was close to $13 \mathrm{kHz}$. As mentioned above, not all spectra are useful for such analysis. At the beginning, the proper frequency band was selected. Figure 3 shows the result of sound sources localization. This map was obtained for the full frequency range. The maps 
presented in Figures 4-6 were obtained for the same time signal but different frequency bands were taken into account. Based on these results the band $3-10 \mathrm{kHz}$ was selected for all subsequent analysis. Lower $(<3 \mathrm{kHz})$ and higher $(>10 \mathrm{kHz})$ frequencies were filtered.
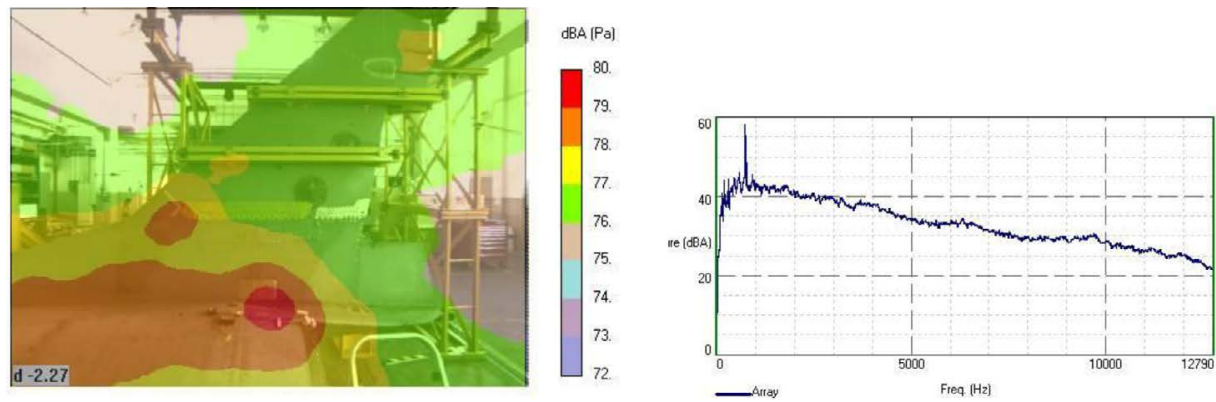

Fig. 3. Localization of sound sources for $0-13 \mathrm{kHz}$ band
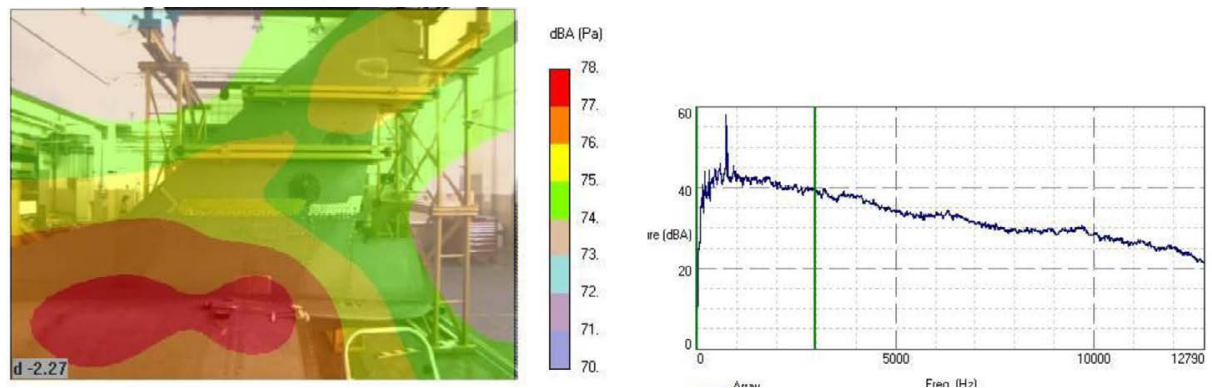

Fig. 4. Localization of sound sources for 0-3kHz band
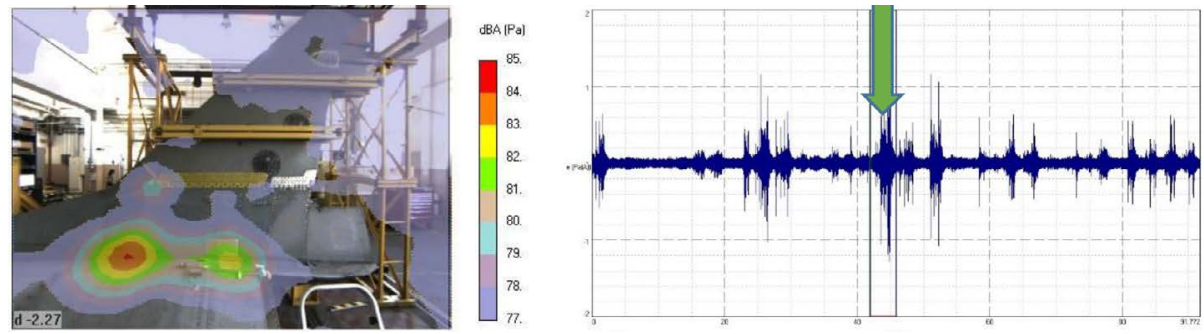

Fig. 5. Localization of sound sources for 3-10k Hz band
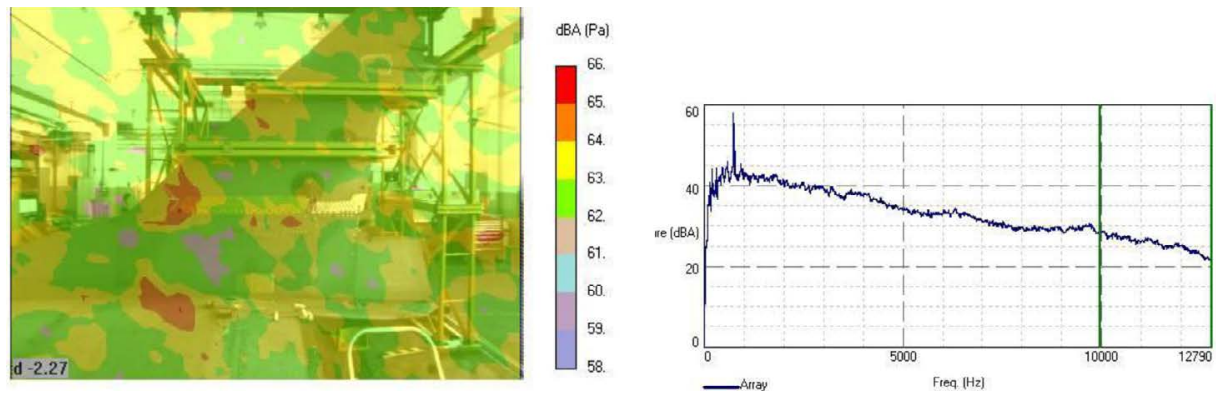

Fig. 6. Localization of sound sources for 10-13 $\mathrm{kHz}$ band 
In the figures below, the analyses of a single time signal are presented. The 60 -second-long signal was recorded but several shorter periods were analyzed separately. The arrows (green) show the exact position of the analyzed time windows on the amplitude vs. time graphs.
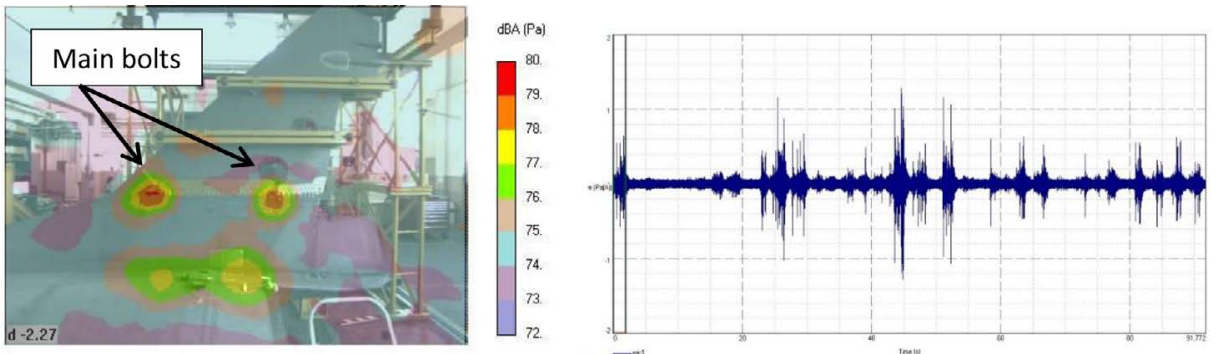

Fig. 7. Localization of sound sources
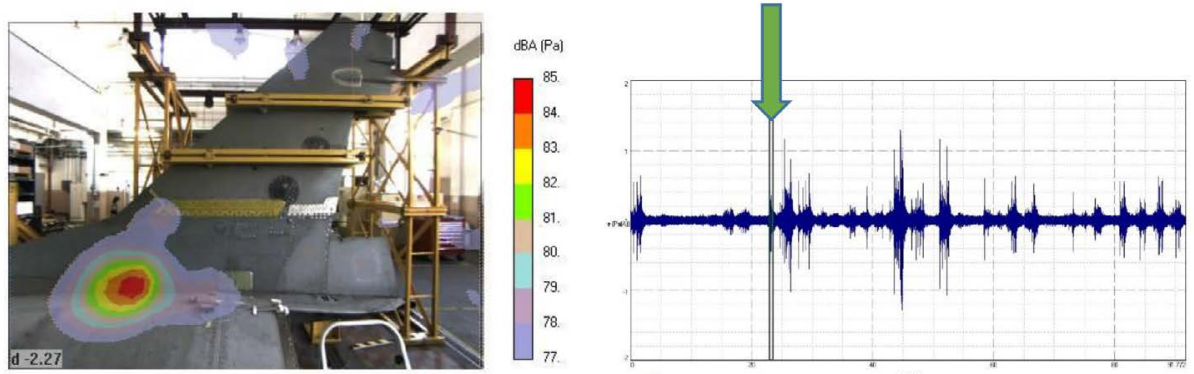

Fig. 8. Localization of sound sources
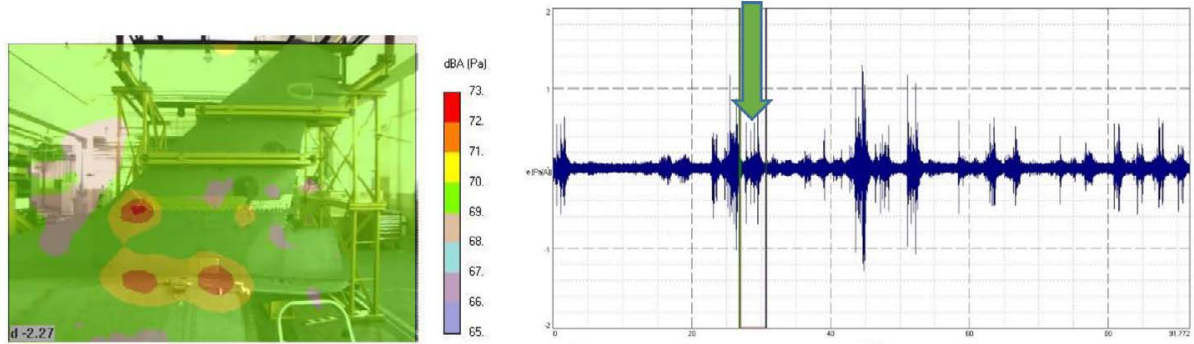

Fig. 9. Localization of sound sources
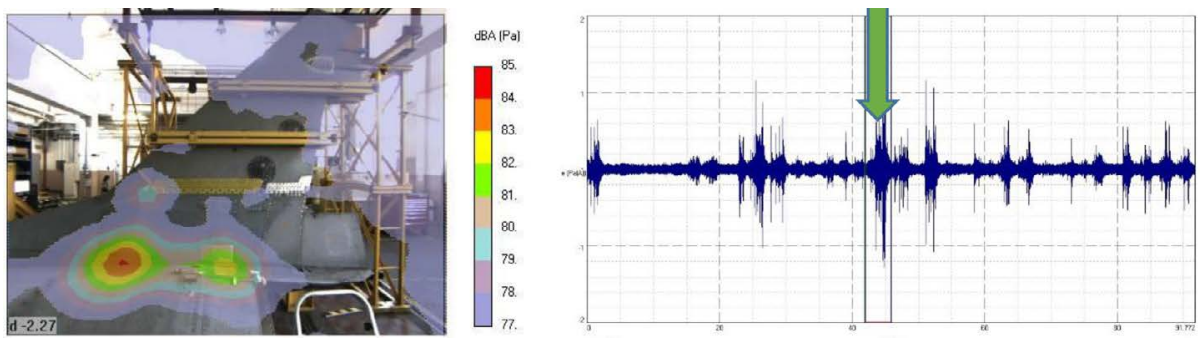

Fig. 10. Localization of sound sources 

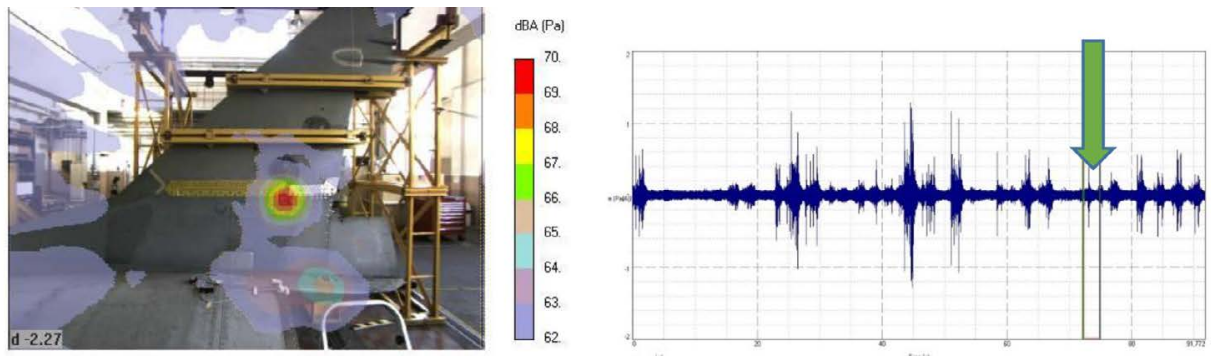

Fig. 11. Localization of sound sources
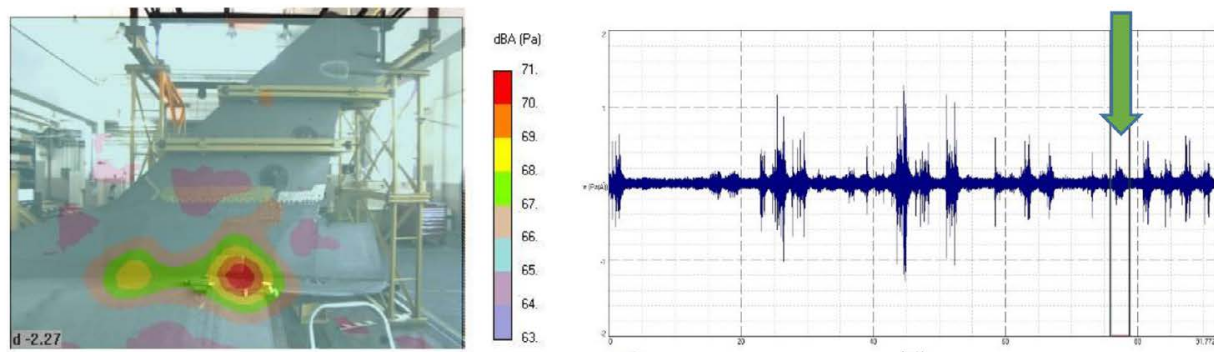

Fig. 12. Localization of sound sources
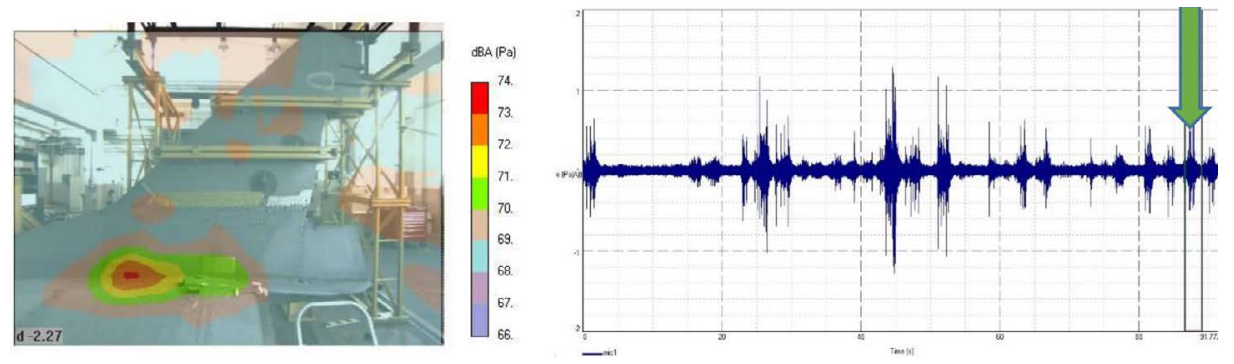

Fig. 13. Localization of sound sources
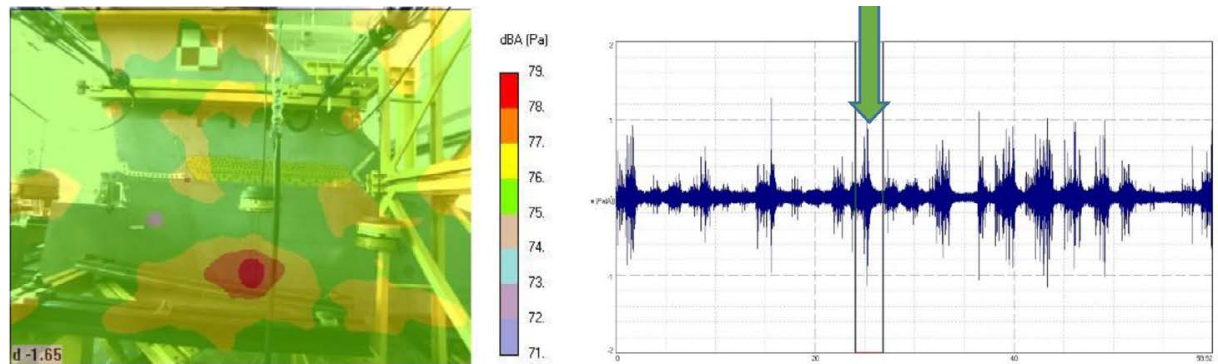

Fig. 14. Localization of sound sources 

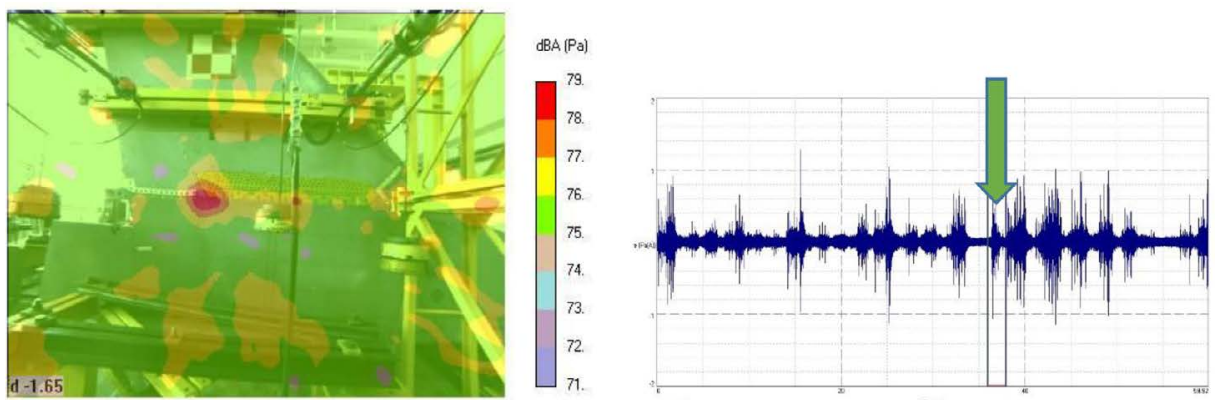

Fig. 15. Localization of sound sources
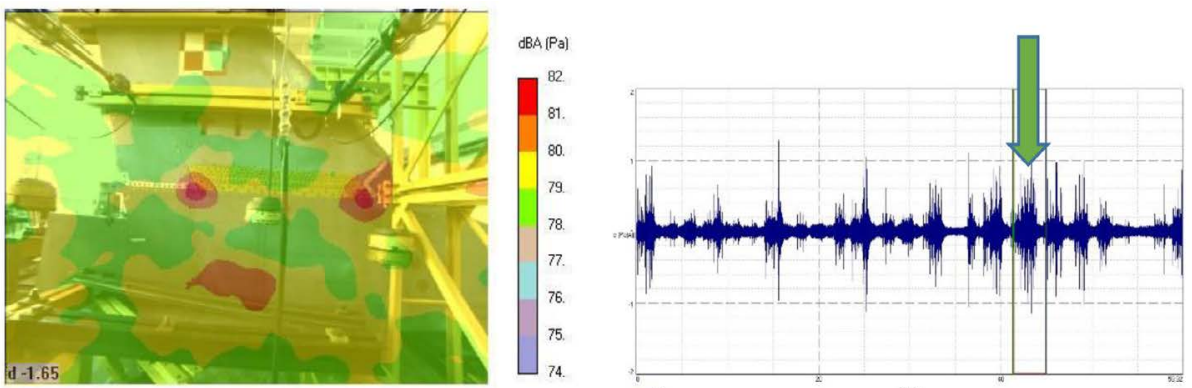

Fig. 16. Localization of sound sources

Figures 7-14 present localization of sound sources on the left side of the stabilizer while Figures 14-17 present the right side view. Based on the presented images, four sound sources can be easily identified on the left side of the stabilizer. Two of them were localized in the connection between the stabilizer and fuselage (see figures 7,9,10,11,13 and 14). The other two were discovered in the connection between the lower and upper parts of the stabilizer (see figures 8,9 and 12). These localizations are also visible from the right side of the stabilizer (see figure 16 and 17).

\subsection{Findings}

The above examples proved that Acoustic Beamforming can be used for localizing sound sources during a full scale fatigue test. The characteristic for FSFT noises have their unique localization. How important can it be? It is a new NDT technique? Definitely not. Not all sound sources indicate damage during FSFT. Nevertheless, such information can be useful. For example, sound indications from the main bolts (Fig .7) may suggest potential problems in the area of bolted joints. In this study, the NDT inspection found loose screws in the connections between the upper and lower stabilizer parts but no cracks. 
After finishing the FSFT the stabilizer will be examined during a teardown NDT inspection. This inspection is likely to define the sound sources, especially those in the connection between the stabilizer and the fuselage.

\section{REFERENCES}

[1] Blacodon D., Caplot M., Élias G.: A source localization technique for helicopter rotor noise. AIAA-1987-2743, 11th Aeroacoustics Conference, Sunnyvale, CA, Oct 19-21, 1987.

[2] Guérin S., Michel U.: Aero-engine noise investigated from flight tests, 12th AIAA/ CEAS Aeroacoustics Conference, Cambridge, Massachusetts (USA), 8-10 May 2006, AIAA-2006-2463.

[3] https://www.plm.automation.siemens.com/global/en/.

[4] Jiang W. K. et al: Research on Diagnosing the Gearbox Faults Based on Near Field Acoustic Holography Journal of Physics, Conference Series 3050120252011.

[5] Maynard J. D., Williams E. G., Lee Y.: Nearfield acoustic holography: I. Theory of generalized holography and the development of NAH. The Journal of the Acoustical Society of America 78, 1395 (1985); https://doi.org/10.1121/1.392911.

[6] Niepokólczycki A., Leski A., Dragan K.: Review of Aeronautical Fatigue Investigations in Poland (2013-2014), Fatigue of Aircraft Structures Vol. 1 (2016) 5-48.

[7] Reymer P., Leski A., Zieliński W., Jankowski K.: The concept of a full scale fatigue test of a SU-22 fighter bomber, Fatigue of Aircraft Structures: Vol. 1 79-87, 2014.

[8] Sijtsma P.: Acoustic beamforming for the ranking of aircraft noise. National Aerospace Laboratory Report NLR-TP-2012-137. https://reports.nlr.nl//xmlui/bitstream/handle/10921/482/TP-2012-137.pdf?sequence=1. 